\title{
A negative feedback loop between XBP1 and Fbw7 regulates cancer development
}

Unbin Chae ${ }^{1}$, Heejin Lee ${ }^{1,2}$, Bokyung Kim ${ }^{1,3}$, Haiyoung Jung ${ }^{4}$, Byeong Mo Kim ${ }^{5}$ Ann- Hwee Lee ${ }^{6}$, Dong-Seok Lee ${ }^{1}$ and Sang-Hyun $\mathrm{Min}^{2}$

\begin{abstract}
In cancer, activation of X-box binding protein (XBP1) has a critical role in tumorigenesis and cancer progression. Transcriptional regulatory mechanism of XBP1 in cancer development has been well known, however, regulation of ubiquitination and degradation of XBP1 has not been elucidated yet. Here we show that Fbw7, a substrate recognition component of the SKP1-Cullin-F-box-type E3 ligase, interacts with XBP1 in a phosphorylation-dependent manner, and facilitates XBP1 ubiquitination and protein degradation. Moreover, Fbw7 inhibits oncogenic pathways including NF-KB, AP1, and Myc induced by XBP1. Interestingly, XBP1 negatively regulates transcription of Fbw7 via a feedback mechanism through NF-KB/E2F-1 axis signaling pathway, suggesting that overexpression of XBP1s may contribute to low level of Fbw7 expression in human cancers. Therefore, a negative feedback loop between Fbw7 and XBP1 contributes to the regulation of tumor development and can be an attractive target for novel therapy in cancers.
\end{abstract}

\section{Introduction}

X-box binding protein (XBP1) plays a critical role in regulation of endoplasmic reticulum (ER) homeostasis ${ }^{1}$, and is also closely associated with in tumorigenesis and progression of tumor ${ }^{2}$. XBP1s, an active form of XBP1, can be translated from mRNA spliced by the ER stress sensor inositol-requiring enzyme $1 \alpha$ (IRE1a) and regulates multiple target genes such as genes associated with cell proliferation and survival ${ }^{3}$. XBP1s is activated in several cancer cells and activated XBP1s promotes breast cancer progression and metastatic capacity ${ }^{4}$. Overexpression of $\mathrm{XBP} 1 \mathrm{~s}$ in cancer cells induces drug resistance by regulating cell cycle and apoptosis genes ${ }^{5}$. A recent study has reported that constitutive XBP1s expression promotes tumorigenesis by controlling anti-tumor immunity in dendritic cells ${ }^{6}$. Therefore, proper regulation of XBP1 expression is important for tumor suppression.

\footnotetext{
Correspondence: Dong-Seok Lee (lee1@knu.ac.kr) or Sang-Hyun

Min (shmin03@dgmif.re.kr)

'School of Life Sciences and Biotechnology, BK21 Plus KNU Creative

BioResearch Group, Kyungpook National University, Daegu, Republic of Korea

${ }^{2}$ Drug Development Center, DGMIF, Daegu, Republic of Korea

Full list of author information is available at the end of the article.
}

However, active form of XBP1, XBP1s, is unstable under common condition. It can be degraded by proteasomes ${ }^{7}$. Therefore, regulation of XBP1s degradation might be able to modulate tumorigenic capacity and tumor progression. Many researches have already demonstrated that transcriptional activation of XBP1 contributes to tumor growth $^{8,9}$. However, the regulation of XBP1s ubiquitination and degradation in cancer cells has not been elucidated yet.

Fbw7 is a substrate recognition component of the Skp1Cullin-F-box (SCF)-type E3 ligase complex and a wellknown tumor suppressor. Fbw7 exerts tumor suppressor function by promoting the ubiquitination and degradation of various oncoproteins including c-Myc, cyclin E, NOTCH-1, and c-Jun ${ }^{10,11}$. Reduced Fbw7 expression and loss-of-function mutations have been demonstrated in various types of human cancer, leading to chromosomal instability and tumorigenesis ${ }^{12}$. Our previous study has shown that Pin1 isomerase can act as an upstream negative regulator of Fbw7 by governing Fbw7 stability and tumor suppressor function ${ }^{13}$. Pin1 interacts with Fbw7 in a phosphorylation-dependent manner. It promotes Fbw7 self-ubiquitination and protein degradation, 
resulting in the amplification of oncogenic pathways. Thus, Pin1-mediated inhibition of Fbw7 is a key signaling pathway that regulates the stability of various oncoproteins in cancer. Our previous study has also demonstrated that Pin1 regulates XBP1 that has a critical role in cancer signaling ${ }^{9}$. Therefore, we speculate that there are regulatory mechanisms of between XBP1 and Fbw7.

Regulation of transcriptional activation of XBP1 plays a critical role in cancer progression and development. However, at post-translational level, the ubiquitination or degradation of XBP1 has not been well investigated. As XBP1 has also putative Fbw7 consensus sequence, the objective of this study was to determine whether Fbw7 might have a possible role in the regulation of ubiquitination and degradation of XBP1. Furthermore, we investigated the effect of XBP1 on Fbw7 regulatory mechanisms as a feedback loop.

\section{Results}

Fbw7 interacts with XBP1 in a phosphorylation-dependent manner

It is known that Fbw7, a substrate recognition component of SCF-type E3 ligase complex, is involved in the ubiquitination and degradation of target proteins ${ }^{13}$. XBP1 has putative Fbw7 consensus sequence in amino acid sequences. Thus, we investigated whether XBP1 might be regulated by Fbw7. Notably, we observed an interaction between XBP1 and Fbw7 based on coimmunoprecipitation (Fig. 1a, b). Furthermore, we detected that XBP1 and Fbw7 also interacts in endogenous level (Fig. 1c). This interaction was eliminated by dephosphorylation of XBP1 with calf intestinal alkaline phosphatase (CIP) and $\lambda$-phosphatase treatment. (Fig. 1d, e), indicating that Fbw7 could bind to XBP1 in a phosphorylation-dependent manner while XBP1 might be a substrate of the Fbw7 E3 ligase complex. It is known that Fbw7 can bind to specific consensus sequences and that Fbw7 recruitment is promoted by phosphorylation ${ }^{14}$. Thus, we selected a putative Fbw7-binding degron motif on Xbp1 (Fig. 2a) and the putative degron motif on XBP1s at $\mathrm{Ser}^{212}$ and $\mathrm{Ser}^{217}$ is conserved in various species (Fig. 2b). After substituting those serine sites to alanine, XBP1s wild-type and S212/217 A were co-immunoprecipitated with FLAG-Fbw7. Interestingly, the interaction between Fbw7 and mutant S212/217 A XBP1s was decreased compared with that between Fbw7 and wild-type XBP1s (Fig. 2c). These results suggested that the $\mathrm{Ser}^{212 / 217}$ sites of XBP1s are important for binding to Fbw7.

\section{Fbw7 promotes ubiquitination and degradation of XBP1}

A previous study has demonstrated that the expression of $\mathrm{XBP1}$ is increased following treatment with the proteasome inhibitor MG132 ${ }^{9}$. Therefore, we hypothesized that XBP1 degradation was mediated by ubiquitination and proteasomes. First, we investigated whether Fbw7 expression affected the protein stability of XBP1s. Cycloheximide (CHX) chase method was used to detect changes in XBP1s stability. In contrast with control cells $(\mathrm{FBW} 7+/+)$, exogenous XBP1s showed higher and longer expression level in Fbw7-deficient cells (FBW7-/-) (Fig. 3a). Furthermore, endogenous XBP1s also indicated higher and longer expression level in Fbw7-deficient cells (FBW7-/-) than control cells $(\mathrm{FBW} 7+/+)$ (Fig. 3b). This suggests that XBP1s is more stable in Fbw7-deficient cells (FBW7-/-), and that Fbw7 can regulate XBP1s expression and destabilize XBP1. Furthermore, we examined XBP1s ubiquitination by Fbw7 expression and detected increased levels of XBP1 ubiquitination in Fbw7-expressing cells compared to that in control vector-expressing cells (Fig. 3c). In contrast, overexpression of inactive Fbw7 mutants did not affect XBP1 ubiquitination. Additionally, ubiquitination of XBP1 was significantly decreased in Fbw7-depleted cells compared to that in Fbw7 wild-type cells (Fig. 3d). These results indicated that Fbw7 can interact with XBP1 in a phosphorylationdependent manner, and promotes ubiquitination of XBP1, subsequently leading to proteasomal degradation of XBP1s.

\section{Fbw7 downregulates the function of XBP1 inducing tumorigenesis}

Given the important role of Fbw7 in regulating ubiquitination and degradation of XBP1s known to have a critical role in tumorigenesis, we determined whether Fbw7 might affect the cellular function and oncogenic signaling pathways of XBP1s. Our previous study has shown that constitutive activation of XBP1 facilitates several oncogenic signaling pathways including Myc, AP1, and NF- $\mathrm{KB}$ activities ${ }^{9}$ Thus, we evaluated the effects of Fbw7 expression on the increased oncogenic signaling pathways by XBP1s using three luciferase reporters (Myc, AP1, and NF-kB). Activities of these three reporters were increased in XBP1s-expressing cells. However, these increased levels of Myc, AP1, and NF- $\mathrm{BB}$ were significantly decreased by Fbw7 expression in a dosedependent manner (Fig. 4a-c). Consistent with the oncogenic function of XBP1, HCT116 cells expressing XBP1s showed a significantly larger focus size as well as increased number of colonies (Fig. 4d, e). Interestingly, depletion of Fbw7 significantly increased the focus number and size, displaying a higher induction effect in XBP1s-overexpressing cells (Fig. 4d, e). Expression of XBP1s in HCT116 cells affected foci formation, similar to that in Fbw7-depleted cells without XBP1s expression (Fig. 4d, e). Migration assay also supported that XBP1s regulation by Fbw7 affects tumorigenic capacity (Fig. 4f, g). In the absence of Fbw7, XBP1s-expressing HCT116 cells significantly promote cancer cell migration compared to HCT116 Fbw7 + / + cells expressing XBP1s (Fig. $4 \mathrm{f}, \mathrm{g})$. Cell proliferation assay data also supported that 


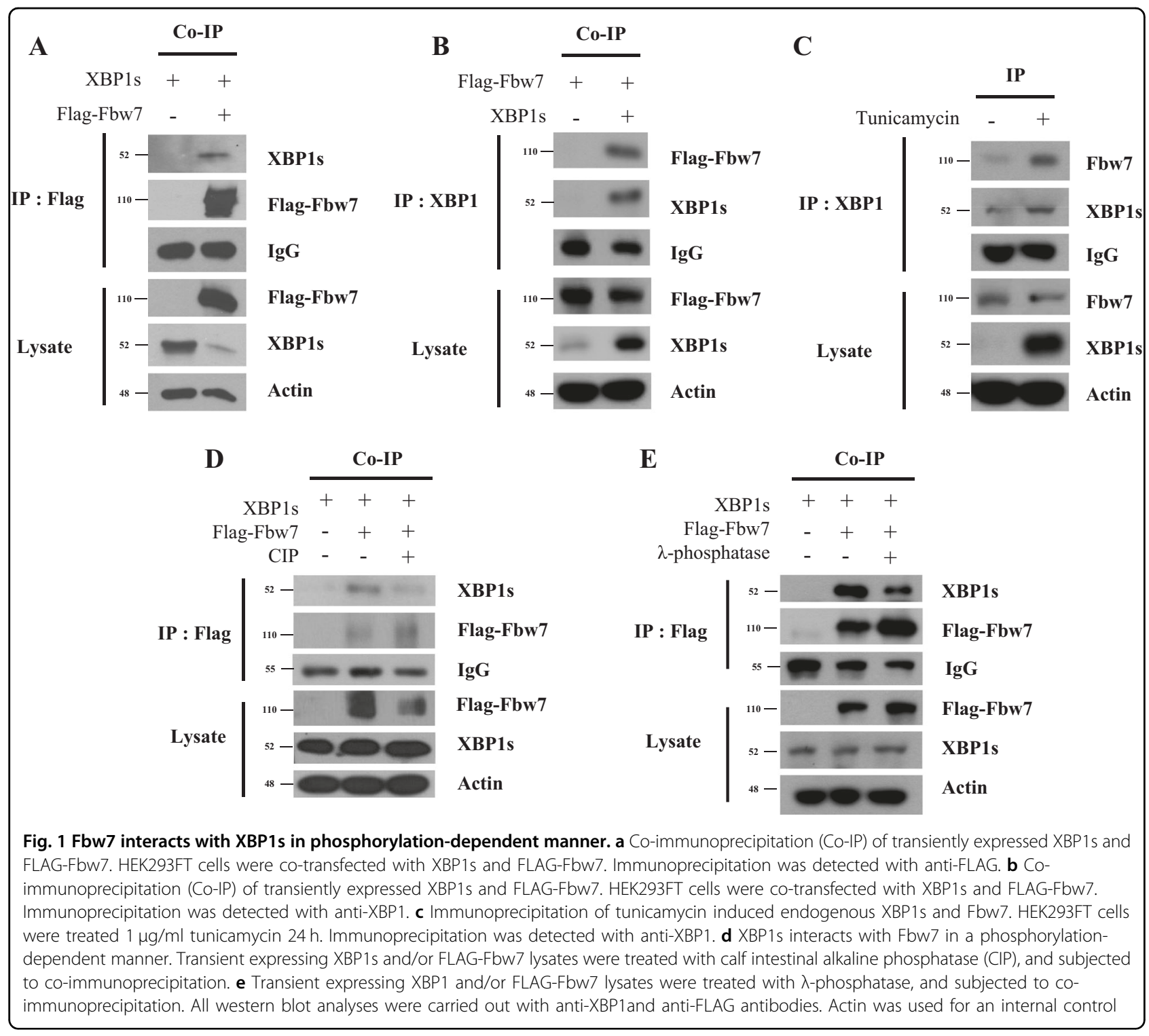

increased XBP1s expression elevates cell proliferation in Fbw7 deficient condition (Fig. 4h). These results together demonstrate that tumorigenic pathways and cellular function of XBP1 are regulated by Fbw7 by reducing the cell transformation capacity of XBP1.

\section{XBP1s downregulates Fbw7 transcription through NF-KB and E2F-1 pathway as a negative feedback mechanism}

In addition, we tested the effect of XBP1s on endogenous or exogenous Fbw7 expression. Various concentrations of XBP1s expression plasmid in HCT116 cells were transfected with FLAG-Fbw7 vector to evaluate the expression of exogenous Fbw7 (Fig. 5a-c). Level of exogenous Fbw7 mRNA was not affected by XBP1s. However, the level of endogenous Fbw7 mRNA level was decreased by XBP1s expression in a dose-dependent manner (Fig. 5d-f). Results of western blot indicated that XBP1s expression had a similar effect on Fbw7 expression (Fig. 5g, h). Expression of endogenous XBP1s induced by Tunicamycin also downregulates endogenous Fbw7 mRNA expression (Fig. 5i). These results suggest that $\mathrm{XBP} 1$ s can downregulate endogenous Fbw7 at the transcriptional level, thereby resulting in the reduction of Fbw7 protein expression.

We next examined how XBP1s regulated Fbw7 endogenous mRNA level in cells. It has been reported that NF$\mathrm{kB}$ (p50) can downregulate the transcription of Fbw7 by inhibiting E2F-1 transcription factor which activates Fbw7 mRNA transcription ${ }^{15}$. Therefore, we hypothesized that $\mathrm{XBP} 1 \mathrm{~s}$ can decrease the Fbw7 transcription level by elevating NF- $\mathrm{kB}$ activation (nuclear localization of $\mathrm{p} 50$ ). Thus, we tested whether XBP1s affects NF-kB activation 


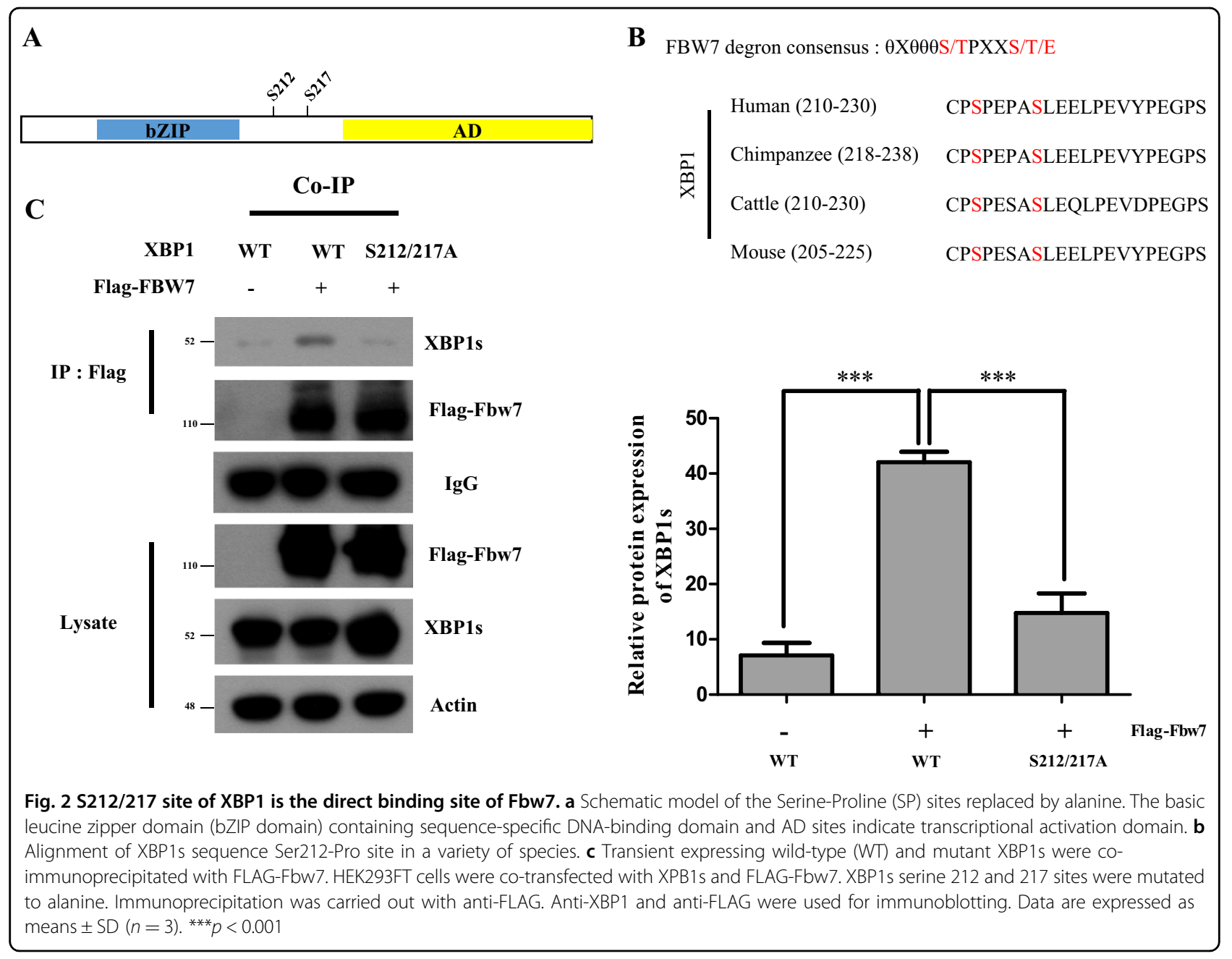

(nuclear localization of p50) and E2F-1 expression. Surprisingly, we found that the amount of p50 localized into nucleus from cytosol was increased in XBP1s overexpressing cells (Fig. 6a, b). Immunocytochemistry data also showed increased localization of p50 into nucleus in XBP1s over-expressing cells (Fig. 6c, d). Furthermore, $\mathrm{XBP} 1 \mathrm{~s}$ expression downregulated E2F-1 expression and E2F-1 luciferase reporter activity upon activation of Fbw7 promoter (Fig. 6e, f). These data suggest that XBP1s can downregulate transcription of Fbw7 through NF- $\mathrm{kB} / \mathrm{E} 2 \mathrm{~F}-$ 1 regulatory pathway (Fig. $6 \mathrm{~g}$ ).

\section{Discussion}

Accumulating researches have revealed that ER-stress plays a critical role on growth and survival of tumor cells $^{16}$. XBP1 is one of the most important ER-stress related gene that are involved in tumorigenesis and metastasis of tumor cells ${ }^{2,17}$. Especially, it has been reported that the active form of XBP1 derived from splicing of XBP1 mRNA is associated with cancer development ${ }^{2}$. However, ubiquitination or degradation of XBP1s at the post-translational level has not been elucidated yet. Here we reported that XBP1s can be regulated by ubiquitination and proteasomal degradation via Fbw7 E3 ubiquitin ligase complex. We founded that Fbw7 interacts with XBP1 directly in a phosphorylation-dependent manner and elevates its ubiquitination and subsequent degradation. As a result, Fbw7 inhibited the ability of $\mathrm{XBP} 1 \mathrm{~s}$ that increases the activities of NF- $\mathrm{KB}, \mathrm{AP} 1$, and Myc signaling pathways. AP-1 and Myc are known transcription factors and oncoproteins ${ }^{18,19}$. Activation of NF$\mathrm{KB}$ controls stress responses in cancer cells ${ }^{20}$ and cytokine induction in immune cells ${ }^{12,21}$. Therefore, modulation of AP1, Myc, and NF-kB activation through Fbw7-XBP1s axis pathway might affect the progression of cancers and immune response from various stress in humans.

In a previous study, we have reported that Pin 1 interacts with Fbw7 in a phosphorylation-dependent manner and promotes Fbw7 self-ubiquitination and protein degradation, indicating that Fbw7 protein destruction and tumor suppressor function are negatively regulated by $\operatorname{Pin} 1^{13}$. Our previous study has also demonstrated that there is a 


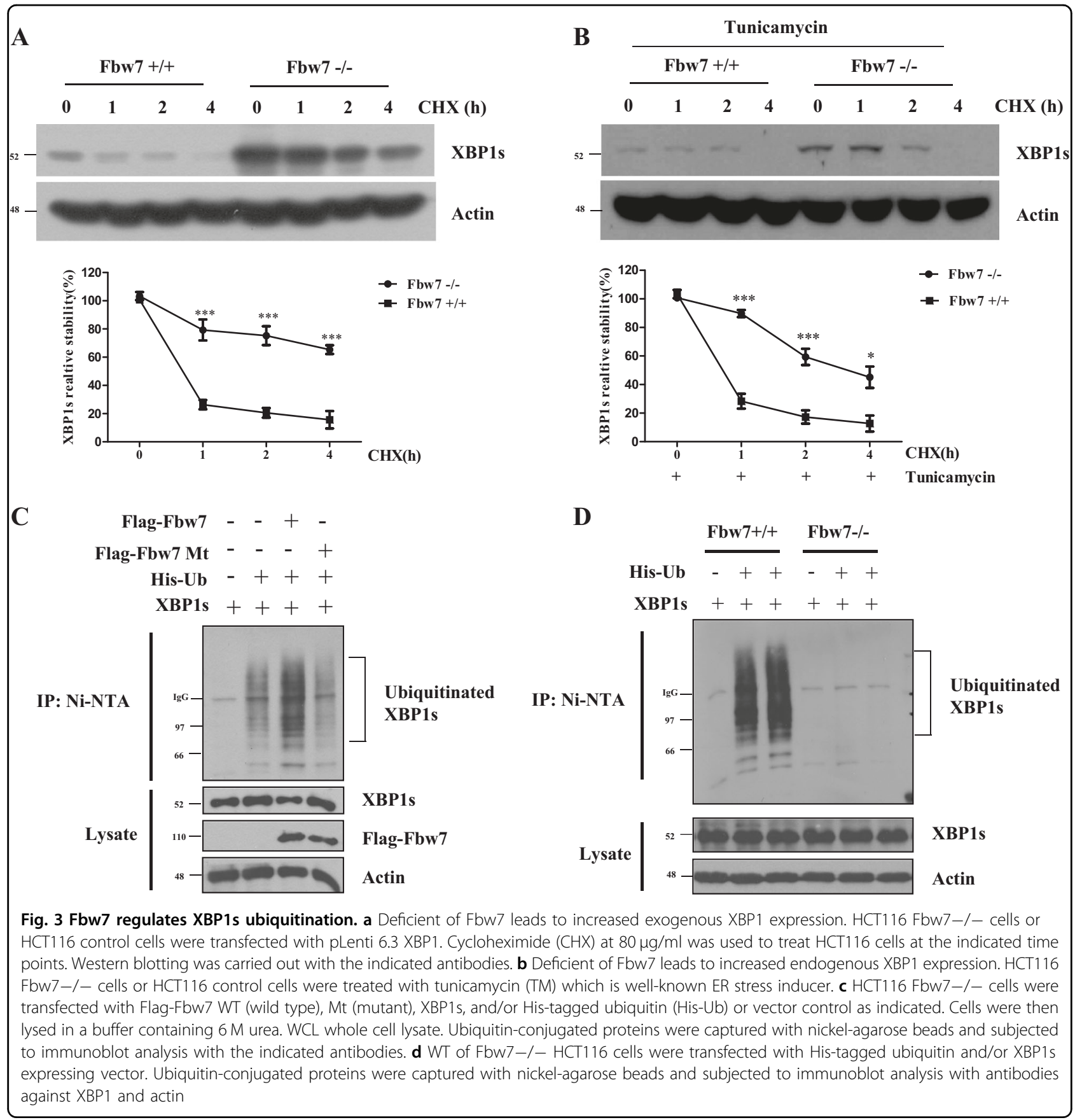

negative feedback mechanism between Pin1 and XBP1s through $\mathrm{p} 53^{9}$. A recent study has suggested that NF- $\mathrm{kB}$ p50 downregulates fbw7 mRNA level by inhibiting E2F-1mediated promoter activation ${ }^{15}$. In the present study, we found that overexpression of XBP1s downregulates transcriptional level of Fbw7 through NF-kB and E2F-1 pathway. Collectively, our results provide a new Pin1Fbw7-XBP1 signal network including Fbw7-mediated blockage of XBP1s ability to enhance AP1, Myc, and
NF- $\mathrm{KB}$ activities and a negative feedback mechanism between Fbw7 and XBP1s during tumorigenesis (Fig. 7).

It has been reported that function of Fbw7 serves as a tumor suppressor through negative regulation of oncoproteins in human cancers ${ }^{22}$. Multiple oncoproteins targeted by Fbw7 are undergoing proteasomal degradation through ubiquitination ${ }^{12}$. Therefore, dysregulation of proteolysis by Fbw7 for oncogenic proteins induces promotion of various cancers. Non-functional Fbw7 


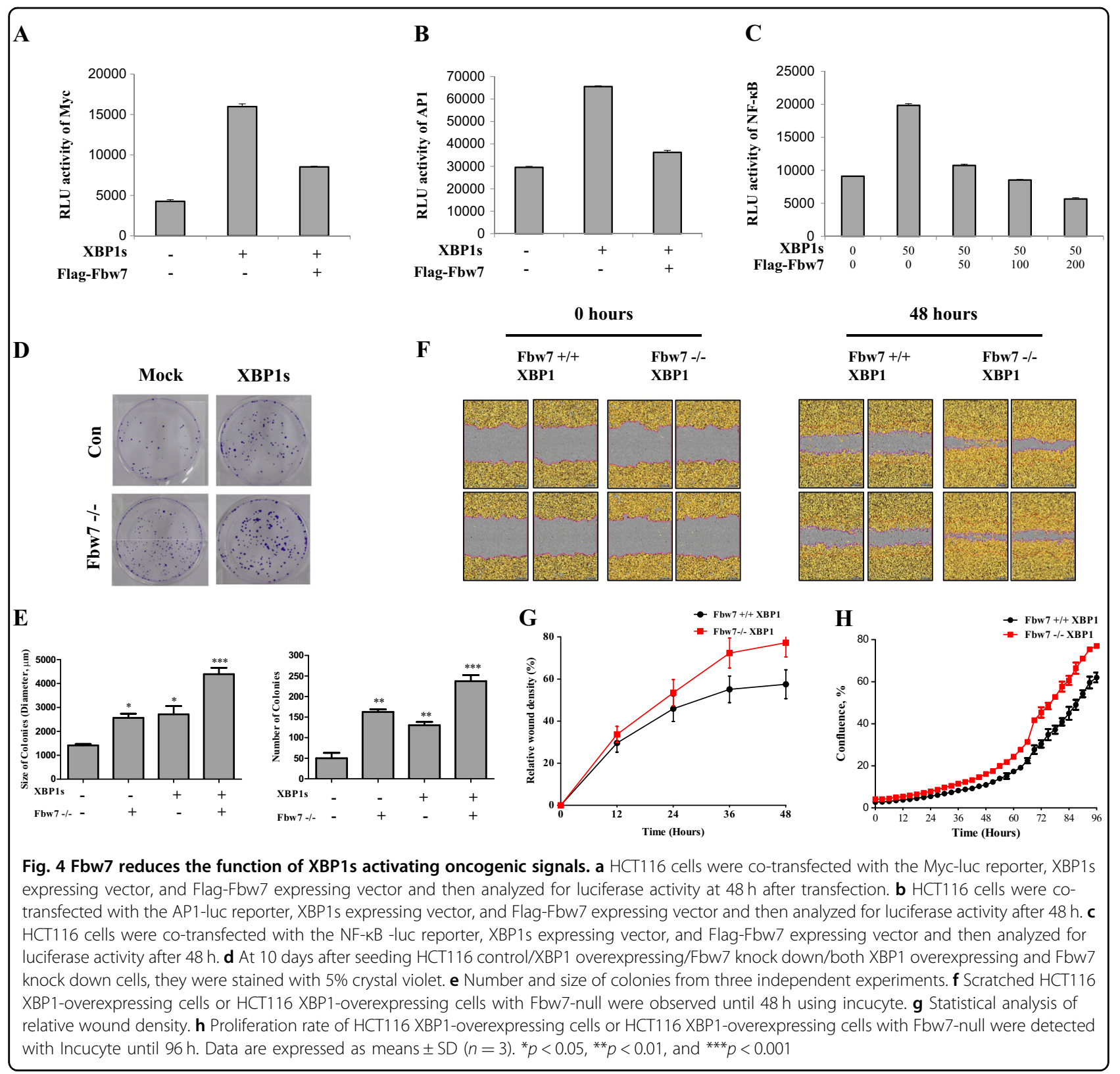

mutations and low expression of Fbw7 have been shown in many human tumors including bladder, endometrial, and colorectal cancers ${ }^{23}$. Due to the critical role of Fbw7 as a tumor suppressor, investigation of regulatory mechanisms related to Fbw7 is still receiving attention. Here we suggested that XBP1 is one of the regulatory substrate of Fbw7. Besides providing the molecular mechanisms for Fbw7-mediated regulation of XBP1s degradation, we showed that upregulation of Fbw7 decreased the ability of XBP1s to enhance cell transformation whereas depletion of Fbw7 increased XBP1s ability. Interestingly, overexpression of XBP1s downregulated the expression of Fbw7 known to have a critical role in suppressing growth and survival of tumor cells. These results implicated that Fbw7 is one of the upstream regulatory proteins for XBP1s signaling. This further suggest that overexpression of XBP1s may contribute to low level of Fbw7 expression, and low level of Fbw7 or dysfunction of Fbw7 by mutation may contribute to high level of XBP1 expression in human cancers.

In the present study, we demonstrated that a reciprocal regulatory mechanism between XBP1 and Fbw7. Our data provide the molecular mechanism of the Fbw7-XBP1 axis that can be used to propose the new pathway about tumorigenesis. We already presented the regulatory mechanisms between Fbw7 and Pin1 or XBP1 and Pin1. 


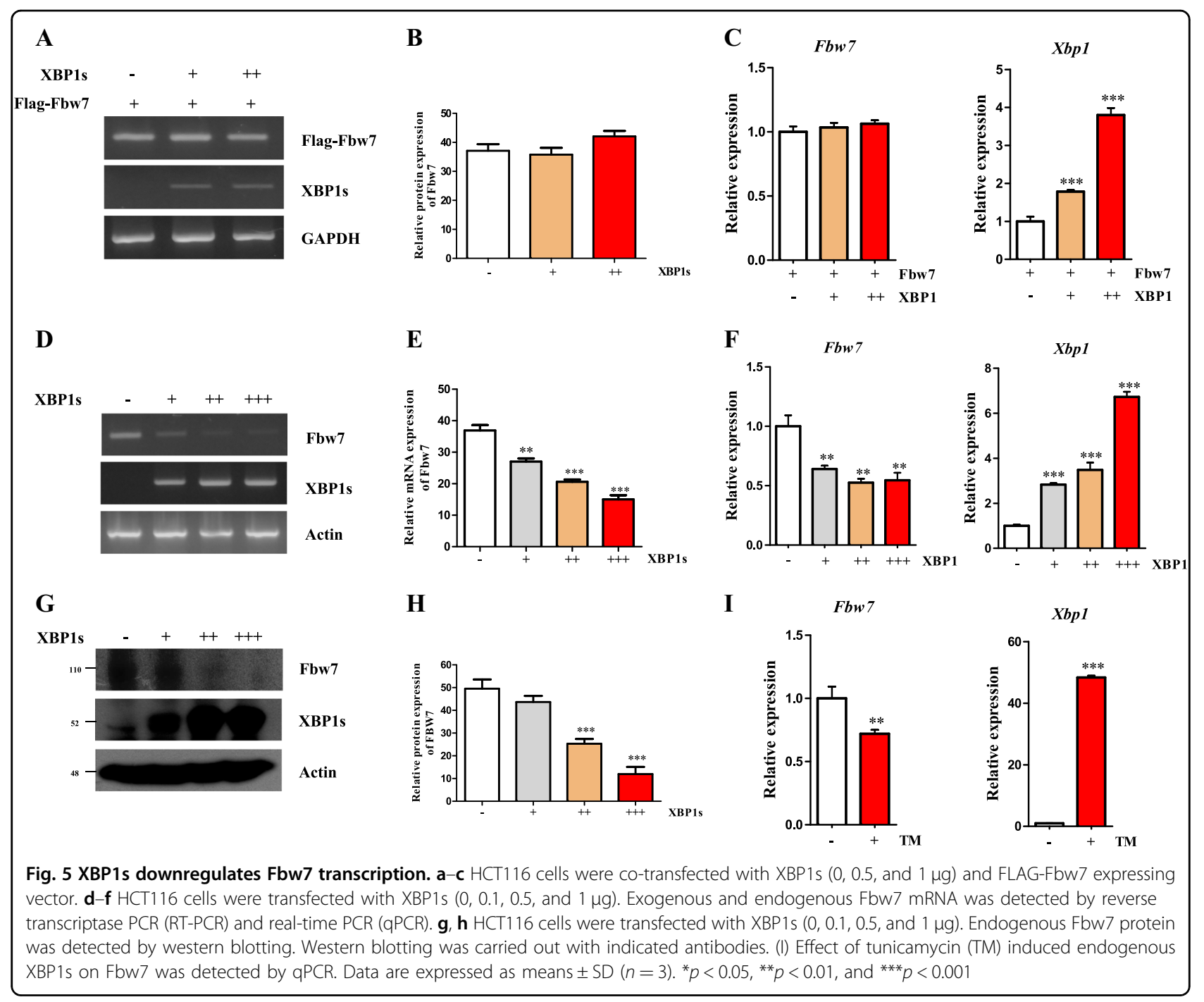

This study suggests a new pathway between XBP1 and Fbw7. Therefore, XBP1, Pin1, and Fbw7 might be closely connected to regulate tumorigenesis. Our findings suggest that the XBP1 and Fbw7 axis might be an attractive target to develop for cancer therapy.

\section{Materials and methods Materials}

Cell culture medium and 1\% penicillin/streptomycin were purchased from Welgene (Daegu, Korea). Fetal bovine serum (FBS) was purchase from Thermo Fisher Scientific (Waltham, MA, USA) and Atlas biologicals (Fort Collins, CO, USA). CHX, CIP and anti-FLAGcoated magnetic beads were purchased from Sigma (St. Louis, MO, USA). All vectors for cloning and transfection, Lipofectamine 3000, and blasticidin were purchased from Invitrogen (Carlsbad, CA, USA).

\section{Cell culture and treatment}

The HEK (human embryonic kindey)-293FT cell line and HCT116 human colon carcinoma cell line were cultured in DMEM (Dulbecco's modified Eagle's medium) containing 10\% FBS and 1\% penicillin/streptomycin. All cells were incubated at $37^{\circ} \mathrm{C}$ in a humidified $5 \% \mathrm{CO}_{2}$ incubator (SANYO, Osaka, Japan). Cell suspensions were treated with $80 \mu \mathrm{M}$ CIP for $30 \mathrm{~min}$ at $37^{\circ} \mathrm{C}$. Cells were transfected transiently with expression plasmids for the protein stability assay. To block the new protein synthesis, cycloheximide was added. Cells were treated with $1 \mu \mathrm{g} / \mathrm{ml}$ tunicamycin for $24 \mathrm{~h}$ at $37^{\circ} \mathrm{C}$.

\section{Cloning and generation of stable cell line}

Coding sequence of XBP1s was amplified by reverse transcription (RT)-PCR with LA Taq polymerase (TaKaRa Bio, Kusatsu, Japan). Amplified XBP1s cDNA was cloned 


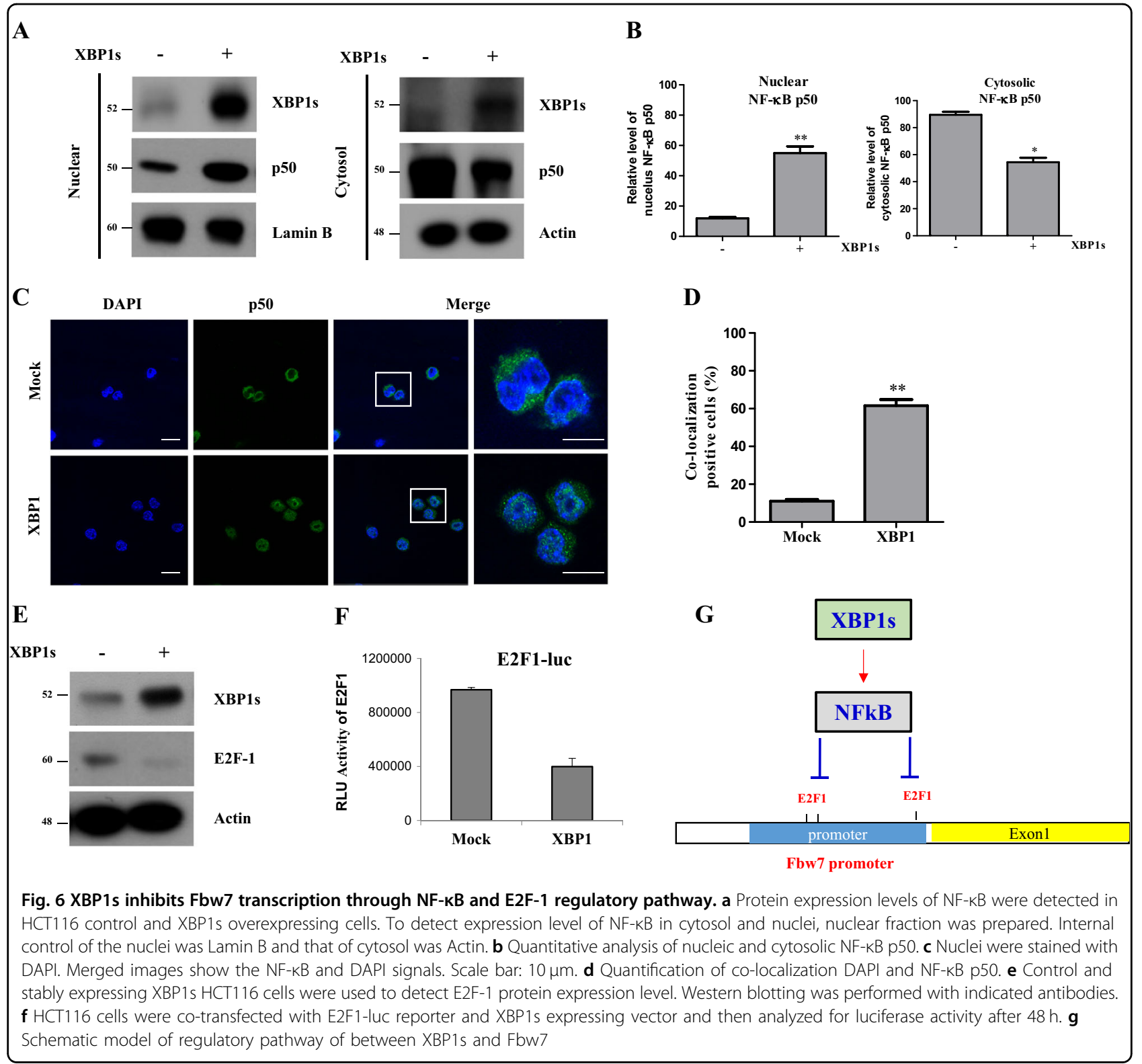

into the $\mathrm{pCR} 8 / \mathrm{GW} / \mathrm{TOPO}$ vector (Invitrogen) to generate pCR8-XBP1. Subsequently, the pCR8-XBP1 vector was subcloned into the pLenti 6.3/V5-DEST vector to generate $6.3 \mathrm{XBP}$. All stable cell lines were generated by transfecting 6.3 XBP1 plasmid into HCT116 and HCT116 Fbw7 knock down cell. XBP-transduced cells were incubated for $72 \mathrm{~h}$ and then selected with blasticidin (Sigma) for 1 week. Gene mutation was performed by point mutagenesis service (Bioneer, Daejeon, Korea).

\section{Immnuoprecipitation and Immnuoblotting}

Relevant proteins were transiently expressed in HEK293FT cells, followed by cell lysis in a buffer as described previously ${ }^{24,25}$. After cell lysis, anti-FLAG-coated magnetic beads (Sigma) or anti-His-tagged agarose beads (Qiagen, Hilden, Germany) were added, followed by further incubation at $4{ }^{\circ} \mathrm{C}$ for $2 \mathrm{~h}$. The precipitated proteins were washed in the same lysis buffer and subjected to immunoblotting with anti-FLAG (1:2000; F3165, RRID: AB_259529) (Sigma), anti-XBP1 (1:2000; 619501, RRID: AB_319507) (Biolegend, San Diego, CA, USA) and anti- $\beta$ actin (1:4000; \#8457, RRID: AB_10950489) (Cell Signaling, Danvers, MA, USA). Cells were also harvested at the indicated time points, and whole-cell-lysates were analyzed by immunoblotting with anti-XBP1 (Biolegend), anti-Fbw7 (1:2000; SC-293423), anti-p50 (NF-kB) (1:2000; SC-8414, RRID: AB_628015), anti-E2F-1 (1:2000; SC-251, RRID: AB_627476) (Santa Cruz Biotechnology, Dallas, 


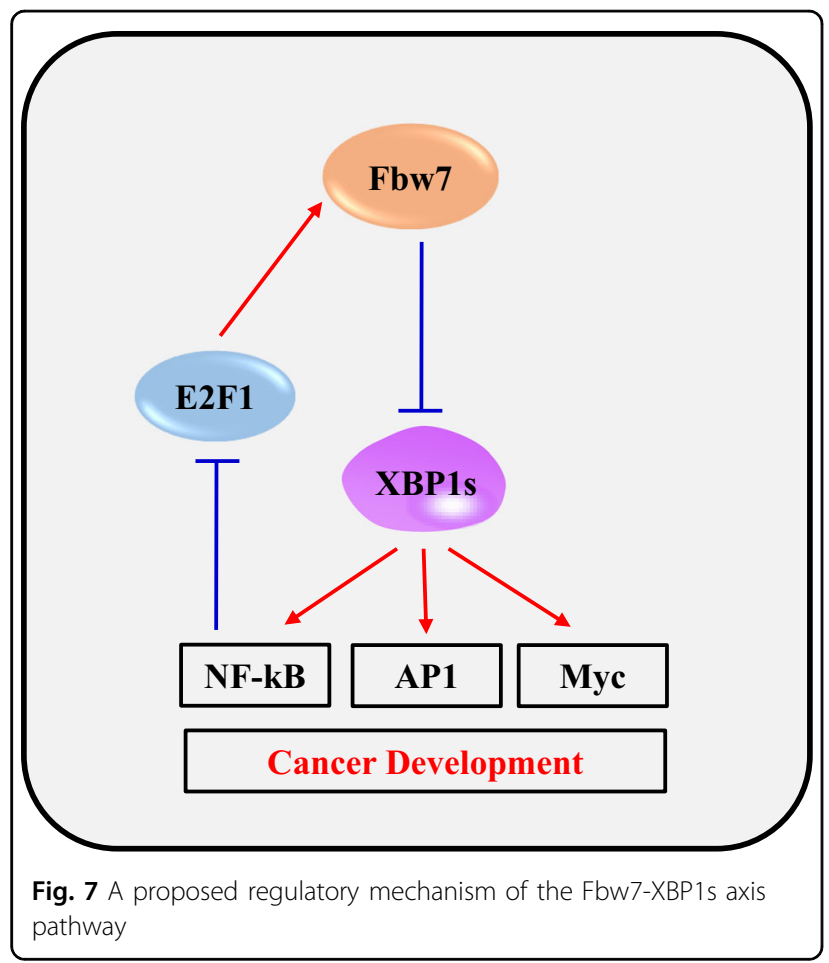

TX, USA), anti- $\beta$-actin (Cell Signaling), and anti-Lamin B (1:2000; PA 50043) (AB Frontier, Seoul, Korea) antibodies.

\section{RNA isolation and RT-PCR/qRT-PCR}

Total RNA was isolated from HCT116 cells using TRIsolution (Invitrogen). cDNA was synthesized from total RNA with reverse transcription premix (Bioneer). PCR was performed using PCR premix (Bioneer). The following PCR primers were used: $5^{\prime}$-XBP1, 5'-AAA CAGAGTAGCAGCGCAGA-3' and $3^{\prime}$-XBP1, 5'TCCTTCTGCGTAGACCTCTGGGAG-3'; and $5^{\prime}$ Fbw7, 5'-CCTAAAGAGTTGGCACTCTA-3' and 3'-Fbw7, 5'ACTCCACCTGTATGTCCCAC-3'; and 5'-GAPDH, 5'ACCACAGTCCATGCCATCAC-3' and $3^{\prime}-\mathrm{GAPDH}, 5^{\prime}-$ TCCACCACCCTGTTGCTGTA- ${ }^{\prime}$. In case of Real-time PCR, the synthesized CDNA was amplified with quantitative real-time PCR (StepOnePlus Real-Time PCR system, Thermo) using FastStart SYBR green Master mix (Roche, Basel, Switzerland) and primers. Transcript level of every gene were normalized with GAPDH and ROX dye was used for experiment control. GAPDH was used as reference gene. The results were presented relative to control using the ddCt method. The following qPCR primers were used: 5'-XBP1, 5'-CCCTCCAGAACA TCTCCCCAT-3' and 3'-XBP1, 5'-ACATGACTGGGTC CAAGTTGT-3'; and 5' Fbw7, 5'-GGCCAAAATGATT CCCAGCAA-3' and 3'Fbw7, 5'-ACTGGAGTTCGTGA CACTGTTA- $3^{\prime}$.

\section{Focus formation}

Stable HCT116 Con, HCT116 XBP1, HCT116 Fbw7-/-, and HCT116 XBP1 Fbw7-/- cell lines were used for focus formation assay as described previously ${ }^{26}$. In Brief, 500 cells were seeded into 6-well plates. At 10 days after seeding, cells were washed twice times with PBS, fixed with $4 \%$ paraformaldehyde (Sigma) and stained with $0.1 \%$ Crystal Violet (Sigma) at room temperature for $1 \mathrm{~h}$. Measuring colony sizes and numbers were carried out by using Image J program.

\section{Scratch wound cell migration assay and cell proliferation}

Cells were plated in 96-well ImageLock ${ }^{\text {tw }}$ tissue culture plate (Essen BioScience, Ann Arbor, MI, USA) at a density of $4 \times 10^{4}$ viable cells per well and grown in medium. After $24 \mathrm{~h}$, the WoundMaker ${ }^{\mathrm{Tm}}$ and wounding procedure to create precise and reproducible wounds in all wells of the 96-well ImageLock $^{\mathrm{mm}}$ plate. After wounding, aspirate the media from each well and PBS wash each well. After washing, add $100 \mathrm{ml}$ of $1 \%$ FBS contained media each well. Remove any bubbles from the assay plate. Place assay plate into the IncuCyte $\mathrm{ZOOM}^{\circ}$ (BioTek, Winooski, VT, USA). Schedule repeat scanning every $2-3 \mathrm{~h}$ for $48 \mathrm{~h}$. Cells (500) were seeded in 96-well plates and cultured in DMEM with $10 \%(\mathrm{v} / \mathrm{v})$ normal FBS for 3 days for measure cell proliferation. The plates were scanned in the IncuCyte imager (Essen Bioscience), and the data were analyzed by the IncuCyte software. Results are representative of three independent experiments.

\section{Luciferase assay}

NF-kB-Luc, Myc-Luc, AP1-Luc, and E2F-Luc reporter plasmids (Promega, Madison, WI, USA) were transfected into cells using Lipofectamine 3000 for the luciferase assay. DNA sample was mixed with $100 \mathrm{ng}$ of CMV-Ren plasmid (Promega) as an internal control and cotrnasfected into cells in 6-well plates. Luciferase assay were performed at $48 \mathrm{~h}$ after transfection using Luciferase Assay Reagent kit (Promega) and a Synergy NEO (BioTek, Winooski, VT, USA). Values obtained were normalized to Renilla luciferase activity.

\section{Nuclear/cytosol isolation and immnuocytochemistry}

Nuclear and cytosol fractions were prepared using a Nuclear and Cytoplasmic Isolation kit (Thermo Scientific). All cells used for immunocytochemistry were fixed with $4 \%$ paraformaldehyde (Sigma) and permeabilized with 0.25\% Trition X-100 in phosphate-buffered saline containing 1\% bovine serum albumin. Fixed cells were incubated with an anti p50 (NF-kB) (1:500; SC-8414, RRID: AB_628015) antibody (Santa Cruz Biotechnology) at $4{ }^{\circ} \mathrm{C}$ overnight. After that, cells were incubated with Alexa 488 goat antimouse secondary antibody (Thermo Scientific) at $4{ }^{\circ} \mathrm{C}$ overnight. Images were obtained using an LSM-710 confocal microscope (Carl Zeiss, Oberkochen, Germany). 


\section{Statistical analysis}

Prism Software (GraphPad Prism version 5.0, La Jolla, CA, USA) was used for all statistical analyses. Data are presented as mean \pm SD of at least three independent experiments $(n \geq 3)$. Dunnett's multiple comparison test was performed for comparisons among groups. A $p$-value of $<0.05$ was considered statistically significant. It is indicated by an asterisk in graphs. $P$-values $<0.01$ and 0.001 are indicated by two and three asterisks, respectively.

\section{Acknowledgements}

This work was supported by the National Research Foundation of Korea (NRF) grant funded by the Korean Government (2013-R1A1A1007596,

2016M3A9E4947 and 2017M3A9E4092542).

\section{Author details}

${ }^{1}$ School of Life Sciences and Biotechnology, BK21 Plus KNU Creative BioResearch Group, Kyungpook National University, Daegu, Republic of Korea. ${ }^{2}$ Drug Development Center, DGMIF, Daegu, Republic of Korea. ${ }^{3}$ Department of Neurology, School of Medicine, UC Davis, Davis, CA 95817, USA. ${ }^{4}$ Immunotherapy Convergence Research Center, Korea Research Institute of Bioscience and Biotechnology, Yuseong-gu, Daejeon 305-806, Republic of Korea. ${ }^{5}$ Severance Integrative Research Institute for Cerebral \& Cardiovascular Diseases (SIRIC), Yonsei University College of Medicine, 50 Yonsei-ro, Seodaemun-gu, Seoul 03722, Republic of Korea. ${ }^{6}$ Department of Pathology and Laboratory Medicine, Weill Cornell Medical College, New York, NY, USA

\section{Conflict of interest}

The authors declare that they have no conflict of interest.

\section{Publisher's note}

Springer Nature remains neutral with regard to jurisdictional claims in published maps and institutional affiliations.

Supplementary Information accompanies this paper at (https://doi.org/ 10.1038/s41389-019-0124-4).

Received: 16 January 2019 Accepted: 28 January 2019

Published online: 19 February 2019

\section{References}

1. Back, S. H., Schroder, M., Lee, K., Zhang, K. \& Kaufman, R. J. ER stress signaling by regulated splicing: IRE1/HAC1/XBP1. Methods 35, 395-416 (2005).

2. Shajahan, A. N., Riggins, R. B. \& Clarke, R. The role of X-box binding protein-1 in tumorigenicity. Drug News Perspect. 22, 241-246 (2009).
3. Mimura, N. et al. Blockade of XBP1 splicing by inhibition of IRE1alpha is a promising therapeutic option in multiple myeloma. Blood 119, 5772-5781 (2012).

4. Chen, $\mathrm{X}$. et al. XBP1 promotes triple-negative breast cancer by controlling the HIF1alpha pathway. Nature 508, 103-107 (2014).

5. Kurata, M. et al. Anti-apoptotic function of Xbp1 as an IL-3 signaling molecule in hematopoietic cells. Cell Death Dis. 2, e118 (2011).

6. Cubillos-Ruiz, J. R. et al. ER stress sensor XBP1 controls anti-tumor immunity by disrupting dendritic cell homeostasis. Cell 161, 1527-1538 (2015).

7. Calfon, M. et al. IRE1 couples endoplasmic reticulum load to secretory capacity by processing the XBP-1 mRNA. Nature 415, 92-96 (2002).

8. He, Y. et al. Emerging roles for XBP1, a sUPeR transcription factor. Gene Expr. 15, 13-25 (2010)

9. Chae, U. et al. Critical role of XBP1 in cancer signalling is regulated by PIN1. Biochem. J. 473, 2603-2610 (2016).

10. Nakayama, K. I. \& Nakayama, K. Regulation of the cell cycle by SCF-type ubiquitin ligases. Semin. Cell Dev. Biol. 16, 323-333 (2005).

11. Cheng, Y. \& Li, G. Role of the ubiquitin ligase Fbw7 in cancer progression. Cancer Metastas-. Rev. 31, 75-87 (2012).

12. Welcker, M. \& Clurman, B. E. FBW7 ubiquitin ligase: a tumour suppressor at the crossroads of cell division, growth and differentiation. Nat. Rev. Cancer $\mathbf{8}$, 83-93 (2008).

13. Min, S. H. et al. Negative regulation of the stability and tumor suppressor function of Fbw7 by the Pin1 prolyl isomerase. Mol. Cell 46, 771-783 (2012).

14. Schulein, C., Eilers, M. \& Popov, N. PI3K-dependent phosphorylation of Fbw7 modulates substrate degradation and activity. FEBS Lett. 585, 2151-2157 (2011).

15. Huang, $H$. et al. NF-kappaB1 inhibits c-Myc protein degradation through suppression of FBW7 expression. Oncotarget 5, 493-505 (2014).

16. Wang, G., Yang, Z. Q. \& Zhang, K. Endoplasmic reticulum stress response in cancer: molecular mechanism and therapeutic potential. Am. J. Transl. Res. 2 65-74 (2010).

17. Xia, T. et al. XBP1 induces MMP-9 expression to promote proliferation and invasion in human esophageal squamous cell carcinoma. Am. J. Cancer Res. $\mathbf{6}$, 2031-2040 (2016).

18. Wagner, E. F. AP-1--introductory remarks. Oncogene 20, 2334-2335 (2001).

19. Denis, N., Kitzis, A., Kruh, J., Dautry, F. \& Corcos, D. Stimulation of methotrexate resistance and dihydrofolate reductase gene amplification by c-myc. Oncogene 6, 1453-1457 (1991).

20. Xia, Y., Shen, S. \& Verma, I. M. NF-kappaB, an active player in human cancers. Cancer Immunol. Res. 2, 823-830 (2014).

21. Lawrence, T. The nuclear factor NF-kappaB pathway in inflammation. Cold Spring Harb. Perspect. Biol. 1, a001651 (2009).

22. Davis, R. J., Welcker, M. \& Clurman, B. E. Tumor suppression by the Fbw7 ubiquitin ligase: mechanisms and opportunities. Cancer Cell 26, 455-464 (2014).

23. Wang, Z. et al. Tumor suppressor functions of FBW7 in cancer development and progression. FEBS Lett. 586, 1409-1418 (2012).

24. Jeong, $K$. et al. p53 negatively regulates Pin1 expression under ER stress. Biochem. Biophys. Res. Commun. 454, 518-523 (2014).

25. Lin, W. C. et al. Endoplasmic reticulum stress stimulates p53 expression through NF-kappaB activation. PLOS ONE 7, e39120 (2012).

26. Hu, R. et al. NF-kappaB signaling is required for XBP1 (unspliced and spliced)mediated effects on antiestrogen responsiveness and cell fate decisions in breast cancer. Mol. Cell. Biol. 35, 379-390 (2015). 